\title{
Pulsation in extremely low-mass helium stars
}

\author{
C. S. Jeffery ${ }^{1}$ and H. Saio ${ }^{2}$ \\ ${ }^{1}$ Armagh Observatory, College Hill, Armagh BT61 9DG, Northern Ireland \\ ${ }^{2}$ Astronomical Institute, School of Science, Tohoku University, Sendai 980-8578, Japan
}

\begin{abstract}
We explore the stability of extremely low-mass stars $\left(M<0.25 M_{\odot}\right)$ across a wide range of composition, effective temperature, and luminosity. We identify the instability boundaries associated with radial oscillations. These are a strong function of both composition and radial order $(0 \leqslant n \leqslant 13)$. The classical blue edge shifts to higher effective temperature and luminosity with decreasing hydrogen abundance. Higher-order modes are more easily excited, and small islands of instability develop. Short-period oscillations have been discovered in the low-mass pre-white dwarf component of the eclipsing binary J0247-25. If its envelope is depleted in hydrogen, J0247-25B is unstable to intermediate-order p modes. Driving is by the classical $\kappa$ mechanism operating in the second helium ionization zone. The observed periods, temperature and luminosity of J0247-25B require an envelope hydrogen abundance $0.2 \leqslant X \leqslant 0.3$.
\end{abstract}

Keywords. stars: early-type, stars: interiors, stars: oscillations, subdwarfs, white dwarfs

White dwarf masses extend from the Chandrasekhar limit to less than $0.17 M_{\odot}$. Extremely low-mass white dwarfs (ELMs) require the prior removal of the hydrogen envelope from a low-mass giant, presumably following interaction in a close binary. Binary systems containing a pre-white dwarf can thus help explain the production of ELMs. Maxted et al. (2011) measured the unseen companion in the bright ecliping binary 1SWASP J024743.37-251549.2 (hereafter J0247-25) to have a mass of $0.23 M_{\odot}$. They identify it as the core of a red giant stripped of its outer envelope before helium ignition. Maxted et al. (2013) show the light curve of J0247-25 to contain variations on timescales of 6-7 and $\approx 40$ minutes, in addition to two eclipses within its $0.668 \mathrm{~d}$ orbital period. They revise the companion mass downward to $0.19 M_{\odot}$. The 40 minute variations are attributed to $\delta$ Scuti-type oscillations in the A-type primary. The multi-periodic $\approx 6-7$ minute oscillations are attributed to p-mode pulsations of order $n \approx 10$ in the pre-white dwarf (J0247-25B). The excitation mechanism for these oscillations is not known.

We (Jeffery \& Saio 2013) have constructed linear non-adiabatic models for the stability of low-mass stellar envelopes against radial pulsation following methods described by Saio et al. (1983) and Jeffery \& Saio (2006a,b) and using the unmodified OPAL tables adopted by Jeffery \& Saio (2007). We considered a range of abundances with hydrogen-mass fraction $X=0.90,0.75,0.50,0.25,0.01$ and 0.002 and a basic metal mass fraction $Z=0.02$. We have assumed the iron and nickel abundances to retain their scaled solar values. A grid of model envelopes was computed for each chemical mixture and for: $M / M_{\odot}=$ $0.18(0.01) 0.25, \log T_{\text {eff }} / \mathrm{K}=3.68(0.02) 4.60$, and $\log \left(L / L_{\odot}\right) /\left(M / M_{\odot}\right)=-0.7(0.1) 3.5$. The first 14 eigenfrequencies were located and stored, including real and imaginary components $\omega_{\mathrm{r}}$ and $\omega_{\mathrm{i}}$, the period $\Pi$ and the number of nodes $n$ in the eigensolution. Modes with $\omega_{\mathrm{i}}<0$ were deemed unstable, i.e. pulsations could be excited. We identified instability regions according to (a) the number of unstable modes in each model and (b) the stability of specific modes. We examined the overall instability boundary for low-order modes up to $n=13$. The results are invariant with the mass in the range $0.18-0.25 M_{\odot}$. We also examined boundaries for individual modes as a function of $\left(T_{\mathrm{eff}}, L / M\right)$ and $X$. These were compared with the properties of J0247-25B. 
For a conventional composition $X=0.75, Z=0.02$ we easily identify the classical instability strip where pulsations due to the second ionisation of helium are excited in the radial, first and second overtone modes $(n=0,1,2)$. The instability strip for $n=$ $0-3$ shifts to the blue and to higher $L / M$ as the hydrogen abundance is reduced, and approaches but does not reach the latest location of J0247-25B at $X=0.002$. The periods of the $n=0-3$ modes at this location are $\approx 700-1000 \mathrm{~s}$, too long to account for the observed periods. For higher order modes, the instability finger for increasing order $n$ extends to lower $L / M$. The blueward drift with decreasing $X$ is replicated. A number of small instability islands develop blueward of the classical blue edge, for example at $X=0.25, \log T_{\text {eff }} \approx 4.2-4.1, \log L / M \approx-0.5-1$, and $X=0.002, \log T_{\text {eff }} \approx 4.15$, $\log L / M \approx 1$. The latest position of J0247-25B (Maxted et al. 2013) lies well within the instability island for $n=8$ radial modes for a hydrogen-poor mixture $X=0.25$ and with exactly the correct period if at least one of the observed modes is a classical radial mode. An $n=9$ mode is also consistent with the observations. Low-degree non-radial modes of the same radial order may equally be excited.

To understand the excitation mechanism, we use a work integral

$$
W(r)=4 \pi \int_{0}^{r} \operatorname{Pr}^{2} \Im\left(\frac{\delta P^{*}}{P} \frac{\delta \rho}{\rho}\right) d r,
$$

where $\delta P$ and $\delta \rho$ are the Lagrangian perturbations of the pressure and density, respectively, $\Im(. .$.$) means the imaginary part, and superscript ' *$ ' means complex-conjugate. For a pulsation mode, layers in which $d W / d r>0(<0)$ contribute to drive (damp) the pulsation. The pulsation is globally excited if $W(R)>0$; i.e. if the work $W$ is positive at the surface. By comparing two models with identical mass, luminosity and effective temperature, but different hydrogen abundances, we find that for J0247-25B the main driving comes from the second helium ionization zone at temperatures around $50000 \mathrm{~K}$, with some help from the first ionization zone, where the opacity derivative $\kappa_{T}=(\partial \ln \kappa / \partial \ln T)_{\rho}$ increases outward; i.e., the $\kappa$ (opacity) mechanism drives the pulsation. In the hydrogen-rich model, the mode is damped because of the high intrinsic opacity of hydrogen. The consequence of reducing this hydrogen-damping is initially to shift the blue-edge to the blue. Although the helium ionization zones are convective, no appreciable energy is carried by convection $\left(L_{\mathrm{rad}} / L \approx 1\right)$.

Intermediate-order p-mode pulsations have been detected in the very low-mass prewhite dwarf J0247-25B (Maxted et al. 2013). Hydrogen-rich stellar envelopes corresponding to the mass, luminosity and effective temperature of J0247-25B are stable against pulsation up to radial order $n=13$. When the hydrogen abundance of the models is reduced, the blue edge of the classical (Cepheid) instability strip becomes bluer. For loworder modes it also becomes narrower. Reduced hydrogen damping allows intermediateorder modes to be excited in regions blueward of this edge. $n=8-10$ pulsations in a $X \approx 0.25$ envelope are completely consistent with the observations of J0247-25B.

\section{Bibliography}

Jeffery, C. S. \& Saio, H. 2006a, MNRAS, 371, 659

Jeffery, C. S. \& Saio, H. 2006b, MNRAS, 372, L48

Jeffery, C. S. \& Saio, H. 2007, MNRAS, 378, 379

Jeffery, C. S. \& Saio, H. 2013, MNRAS, 435, 885

Maxted, P. F. L., Anderson, D. R., Burleigh, M. R., et al. 2011, MNRAS 418, 1156

Maxted, P. F. L., Serenelli, A. M., Miglio, A., et al. 2013, Nature, 498, 463

Saio, H., Winget, D. E., \& Robinson, E. L. 1983, ApJ, 265, 982 